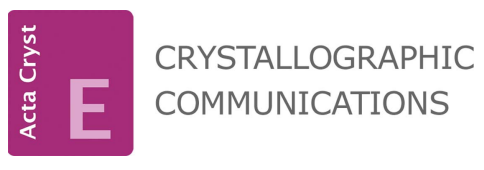

ISSN 2056-9890
Received 26 November 2018

Accepted 7 December 2018

Edited by C. Rizzoli, Universita degli Studi di Parma, Italy

Keywords: halogen chalcone; crystal structure; DFT; Hirshfeld surface; UV-vis; HOMOLUMO; molecular electrostatic potential.

CCDC reference: 1878940

Supporting information: this article has supporting information at journals.iucr.org/e

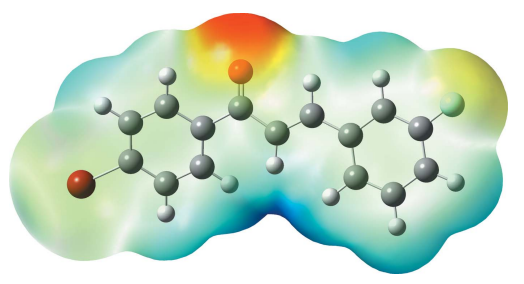

OPEN $\odot$ ACCESS

\section{Crystal structure, Hirshfeld surface analysis and DFT studies of $(E)$-1-(4-bromophenyl)-3-(3-fluoro- phenyl)prop-2-en-1-one}

\author{
Muhamad Fikri Zaini, Ibrahim Abdul Razak, Mohamad Zahid Anis and Suhana \\ Arshad* \\ X-ray Crystallography Unit, School of Physics, Universiti Sains Malaysia, 11800 USM, Penang, Malaysia. \\ *Correspondence e-mail: suhanaarshad@usm.my
}

The asymmetric unit of the title halogenated chalcone derivative, $\mathrm{C}_{15} \mathrm{H}_{10} \mathrm{BrFO}$, contains two independent molecules, both adopting an s-cis configuration with respect to the $\mathrm{C}=\mathrm{O}$ and $\mathrm{C}=\mathrm{C}$ bonds. In the crystal, centrosymmetrically related molecules are linked into dimers via intermolecular hydrogen bonds, forming rings with $R_{1}^{2}(6), R_{2}^{2}(10)$ and $R_{2}^{2}(14)$ graph-set motifs. The dimers are further connected by $\mathrm{C}-\mathrm{H} \cdots \mathrm{O}$ interactions into chains parallel to [001]. A Hirshfeld surface analysis suggests that the most significant contribution to the crystal packing is by $\mathrm{H} \cdots \mathrm{H}$ contacts $(26.3 \%)$. Calculations performed on the optimized structure obtained using density functional theory (DFT) at B3LYP with the 6-311 G++(d,p) basis set reveal that the HOMO-LUMO energy gap is $4.12 \mathrm{eV}$, indicating the suitability of this crystal for optoelectronic and biological applications. The nucleophilic and electrophilic binding site regions are elucidated using the molecular electrostatic potential (MEP).

\section{Chemical context}

Chalcones are natural or synthetic compounds belonging to the flavonoid family (Di Carlo et al., 1999), consisting of openchain flavonoids in which the aromatic rings are linked by a three-carbon $\alpha, \beta$-unsaturated carbonyl system (Thanigaimani et al., 2015). Chalcone derivatives have attracted significant interest in the field of non-linear optics due to their excellent blue-light transmittance, good crystal stability, large nonlinear optical coefficients and relatively short cut-off wavelengths of transmittance (Goto et al., 1991; Patil et al., 2006a,b; Zhao et al., 2000). The presence of halogen substitutions results in alterations of the physicochemical properties and biological activities of organic compounds, without introducing much major steric change. As a result of this, many researchers have worked intensively on fluorine substitution to develop a wide range of biologically active materials (O'Hagan et al., 2008). As part of our studies in this area, fluoro and bromo substituents were introduced in the title compound and the resulting organic molecular crystal is reported herein in term of its structural stability, the percentage contributions of the various interactions to the crystal packing, and electronic charge transfer within the molecule.

\section{Structural commentary}

The asymmetric unit of the title compound [Fig. 1(a)] contains two independent molecules $(A$ and $B$ ) with different conformations: the fluorobenzene group in molecule $A$ is rotated by 
approximately $180^{\circ}$ about the $\mathrm{C} 9-\mathrm{C} 10$ bond with respect to molecule $B$, the $\mathrm{C} 9 \cdots \mathrm{C} 11-\mathrm{C} 12-\mathrm{F} 1$ torsion angle formed by non-bonded atoms being 178.4 (3) and -177.0 (3) ${ }^{\circ}$ in molecules $A$ and $B$, respectively. The optimized structure of the title compound was performed with the Gaussian $09 \mathrm{~W}$ software package (Frisch et al., 2009) using the DFT method at the B3LYP/6-311 G++(d,p) level to provide information about the molecular geometry.<smiles>CC(=O)c1ccc(Br)cc1</smiles><smiles>O=C(/C=C/c1cccc(F)c1)c1ccc(Br)cc1</smiles>

Bond length and angles are unexceptional and fall within the expected ranges. The enone group (O1/C7-C9) of both molecules $A$ and $B$ adopts $s$-cis configurations with respect to the $\mathrm{C} 7=\mathrm{O} 1 \quad[\mathrm{C} 7 A-\mathrm{O} 1 A=1.207$ (4) $\AA$; $\mathrm{C} 7 B-\mathrm{O} 1 B=$ $1.221(3) \AA]$ and $\mathrm{C} 8=\mathrm{C} 9[\mathrm{C} 8 A-\mathrm{C} 9 A=1.321$ (4) $\AA$; $\mathrm{C} 8 B-$ $\mathrm{C} 9 B=1.322(4) \AA]$ double bonds. The values of the bond lengths within the enone group obtained by theoretical study are in good agreement with the results of the X-ray analysis $(\mathrm{C} 7-\mathrm{O} 1=1.223 \AA$; $\mathrm{C} 8-\mathrm{C} 9=1.345 \AA)$. The molecule is essentially planar, the $\mathrm{O} 1-\mathrm{C} 7-\mathrm{C} 8-\mathrm{C} 9$ torsion angle being $1.0(5)^{\circ}$ for molecule $A$ and $3.9(4)^{\circ}$ for molecule $B$. The corresponding torsion angle from the DFT study is $-5.024^{\circ}$. This slight deviation from the experimental value is due to the

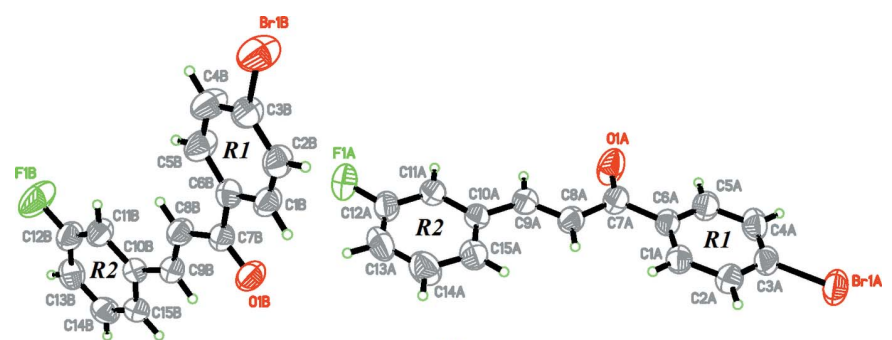

(a)

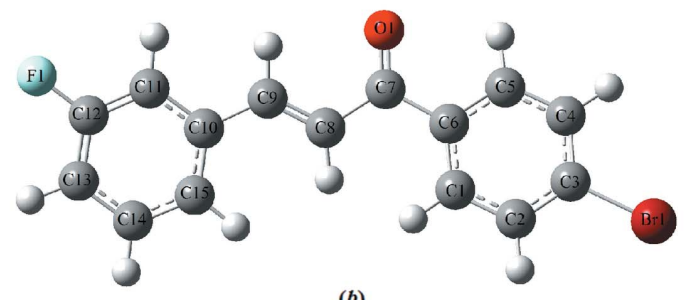

Figure 1

(a) The molecular structure of the title compound with displacement ellipsoids drawn at the $50 \%$ probability level and $(b)$ the optimized molecular structure of the title compound generated using the DFT method at the B3LYP/6-311 G++(d,p) level.
Table 1

Hydrogen-bond geometry $\left(\AA{ }^{\circ}\right)$.

\begin{tabular}{lllll}
\hline$D-\mathrm{H} \cdots A$ & $D-\mathrm{H}$ & $\mathrm{H} \cdots A$ & $D \cdots A$ & $D-\mathrm{H} \cdots A$ \\
\hline $\mathrm{C} 13 A-\mathrm{H} 13 A \cdots \mathrm{O} 1 B$ & 0.93 & 2.52 & $3.427(4)$ & 165 \\
$\mathrm{C} 9 A-\mathrm{H} 9 A A \cdots \mathrm{O} 1 A^{\mathrm{i}}$ & 0.93 & 2.52 & $3.362(4)$ & 151 \\
$\mathrm{C} 11 A-\mathrm{H} 11 A \cdots \mathrm{O} 1 A^{\mathrm{i}}$ & 0.93 & 2.45 & $3.294(4)$ & 151 \\
$\mathrm{C} 15 B-\mathrm{H} 15 B \cdots \mathrm{O} 1 B^{\text {ii }}$ & 0.93 & 2.50 & $3.377(4)$ & 157 \\
\hline
\end{tabular}

Symmetry codes: (i) $-x+1,-y+1,-z+1$; (ii) $-x+1,-y+1,-z+2$.

fact that the optimization is performed in isolated conditions, whereas the crystal environment and hydrogen-bonding interactions affect the results of the X-ray structure (Zainuri et al., 2017). The $\mathrm{C} 1-\mathrm{C} 6(R 1)$ and $\mathrm{C} 10-\mathrm{C} 15(R 2)$ phenyl rings in both molecules are approximately coplanar, the dihedral angle they form being $3.75(15)^{\circ}$ and $5.56(15)^{\circ}$ in molecules $A$ and $B$, respectively. Furthermore, the dihedral angles formed by the mean plane through the enone group [maximum deviation of 0.004 (3) $\AA$ for atoms $\mathrm{C} 7 A / \mathrm{C} 8 A$, and 0.016 (3) $\AA$ for atom $\mathrm{C} 7 B]$ and the $R 1$ and $R 2$ rings are $6.3(2)$ and $2.6(2)^{\circ}$ in molecule $A$, and 6.42 (19) and $4.41(19)^{\circ}$ in molecule $B$.

\section{Supramolecular features}

In the crystal packing of the compound, the $B$ molecules are centrosymmetrically connected via intermolecular $\mathrm{C} 15 B-$ $\mathrm{H} 15 B \cdots \mathrm{O} 1 B$ interactions, forming a ring with an $R_{2}^{2}(14)$ graph-set motif [Table 1, Fig. 2(a)]. Similarly, the intermolecular $\mathrm{C} 9 A-\mathrm{H} 9 A A \cdots \mathrm{O} 1 A$ and $\mathrm{C} 11 A-\mathrm{H} 11 A \cdots \mathrm{O} 1 A$ [Table 1, Fig. 2(b)] hydrogen bonds also connect the $A$ mol-

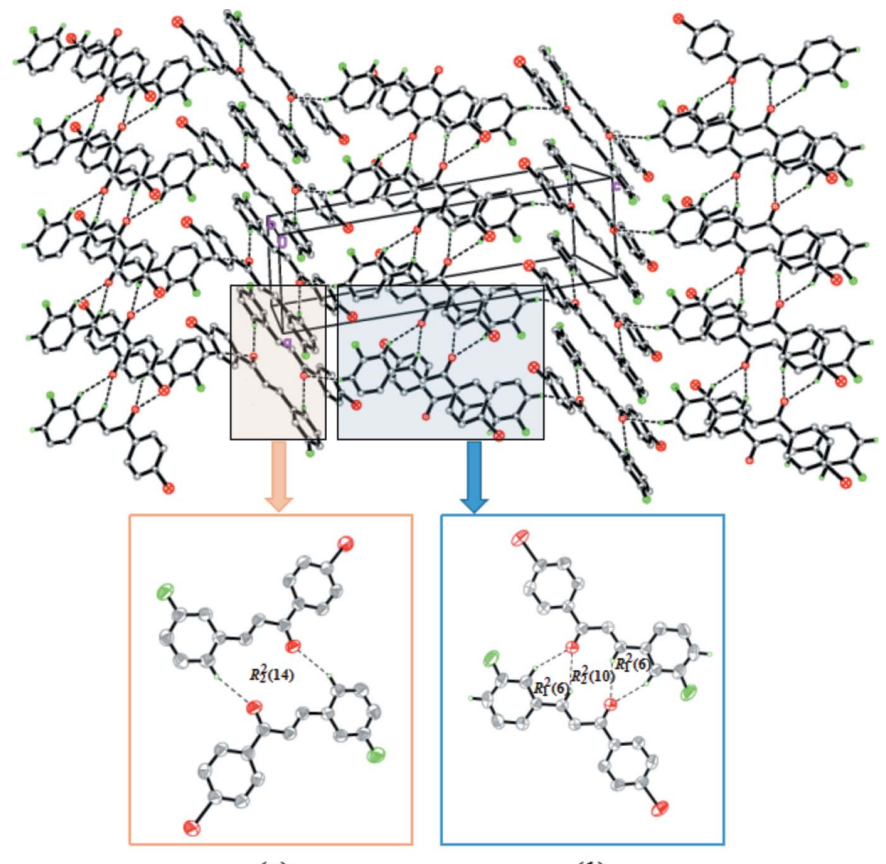

(a)

(b)

Figure 2

Crystal packing of the title compound showing $\mathrm{C}-\mathrm{H} \cdots \mathrm{O}$ hydrogen bonds (dotted lines). $\mathrm{H}$ atoms not involved in hydrogen bonding are omitted. The insets show the formation of $(a) R_{2}^{2}(14)$ ring motifs and $(b)$ $R_{1}^{2}(6)$ and $R_{2}^{2}(10)$ ring motifs. 


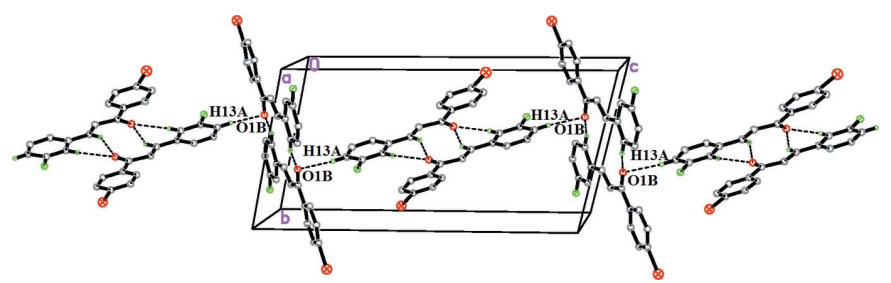

Figure 3

Partial crystal packing of the title compound viewed approximately down the $a$ axis showing the formation of a molecular chain parallel to the $c$ axis by $\mathrm{C}-\mathrm{H} \cdots \mathrm{O}$ interactions (dotted lines).

ecules into inversion dimers, forming two $R_{1}^{2}(6)$ and one $R_{2}^{2}(10)$ ring motifs. Finally, the $\mathrm{C} 13 A-\mathrm{H} 13 A \cdots \mathrm{O} 1 B$ interactions act as a bridge, linking the dimers into chains extending parallel to the $c$ axis (Fig. 3).

\section{Hirshfeld Surface analysis}

Hirshfeld surface analysis provides the percentage contribution of the intermolecular interactions inside the unit-cell packing. The surface and the related two-dimensional fingerprint plots were generated with CrystalExplorer3.1 (Wolff et al., 2012). The $d_{\text {norm }}$ and $d_{e}$ surfaces are presented in Fig. 4(a) and Fig. 4(b), respectively. All $\mathrm{C}-\mathrm{H} \cdots \mathrm{O}$ contacts are recognized in the $d_{\text {norm }}$ mapped surface as deep-red depression areas showing the interaction between the neighbouring molecules [Fig. 4(a)]. Further existence of these contacts can be visualized under the $d_{e}$ surfaces. The side view I (Fig. 4) shows that the $A$ molecules may interact through $\mathrm{C} 9 A-$ $\mathrm{H} 9 A A \cdots \mathrm{O} 1 A$ and $\mathrm{C} 11 A-\mathrm{H} 11 A \cdots \mathrm{O} 1 A$ interactions,

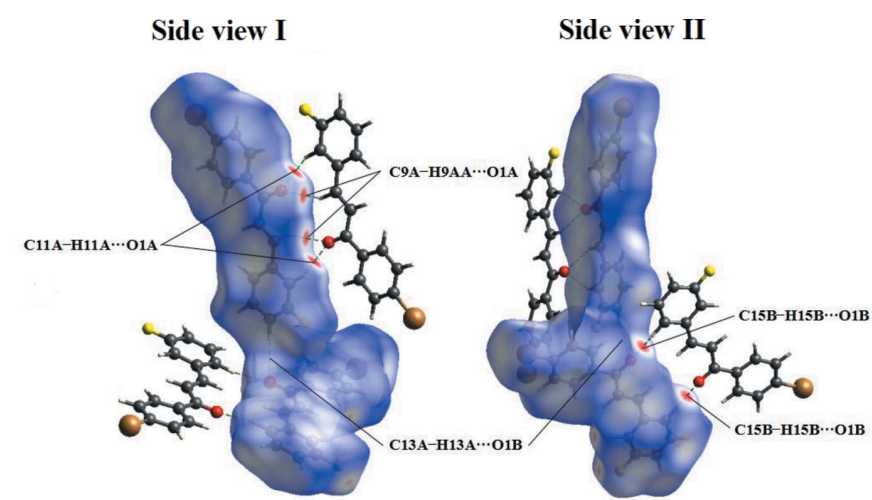

(a)
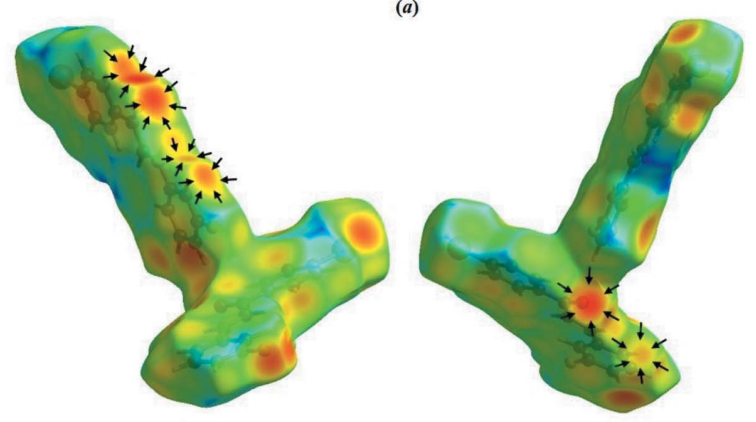

(b)

Figure 4

Hirshfeld surfaces of the title compound mapped over $d_{\text {norm }}$ and $d_{e}$.
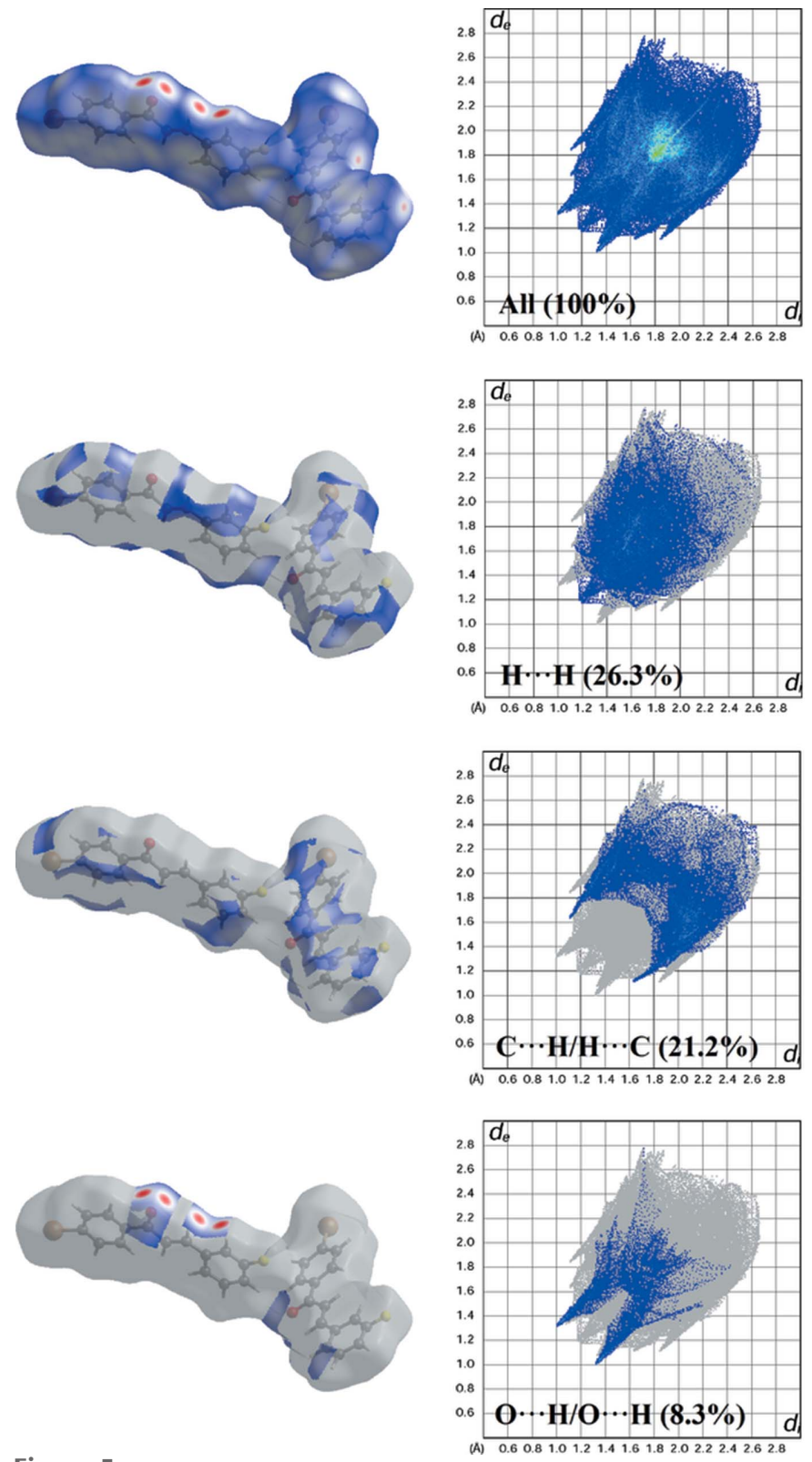

Figure 5

Two-dimensional fingerprint plots with a $d_{\text {norm }}$ view showing the percentage contributions to the total Hirshfeld surface.

resulting in the formation of three ring motifs. Meanwhile, side view II (Fig. 4) indicates that for $B$ molecules only one ring motif is achieved through $\mathrm{C} 15 B-\mathrm{H} 15 B \cdots \mathrm{O} 1 B$ interactions. Two-dimensional fingerprint plots provide information about the major and minor percentage contribution of interatomic contacts in the compound. The blue colour refers to the frequency of occurrence of the $\left(d_{i}, d_{e}\right)$ pair and the grey colour is the outline of the full fingerprint (Ternavisk et al., 2014). The fingerprint plots (Fig. 5) show that the $\mathrm{H} \cdots \mathrm{H}$ contacts clearly make the most significant contribution to the Hirshfeld surface $(26.3 \%)$ : there is one distinct spike with a $d_{e}+d_{i}$ value approximately less than the sum of Van der Waals radii $(2.4 \AA)$. In addition, $\mathrm{C} \cdots \mathrm{H} / \mathrm{H} \cdots \mathrm{C}$ and $\mathrm{O} \cdots \mathrm{H} / \mathrm{H} \cdots \mathrm{O}$ contacts contribute $21.2 \%$ and $8.3 \%$, respectively, to the Hirshfeld surface. In particular, the $\mathrm{O} \cdots \mathrm{H} / \mathrm{H} \cdots \mathrm{O}$ contacts indicate the 


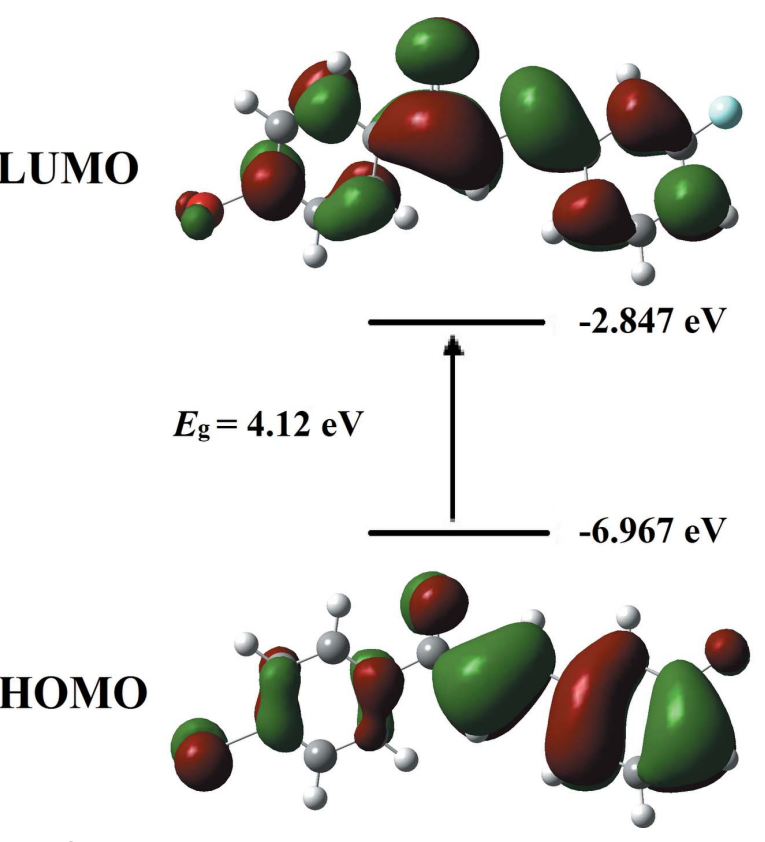

Figure 6

Molecular orbitals showing the HOMO-LUMO electronic transitions in the title compound.

presence of intermolecular $\mathrm{C}-\mathrm{H} \cdots \mathrm{O}$ interactions where the distance is shorter than the sum of $d_{e}+d_{i}(\sim 2.32 \AA)$.

\section{Frontier molecular orbital and UV-vis Analyses}

Frontier molecular orbital analysis is an important tool in quantum chemistry for studying the molecular electronic charge mobility from the highest occupied molecular orbital (HOMO) and the lowest unoccupied molecular orbital (LUMO). The HOMO-LUMO separation confirms the energy gap of the compound where it is responsible for the ICT (intramolecular charge transfer) from the end-capping electron-donor groups to the efficient electron-acceptor groups through the $\pi$-conjugated path. The electron-density

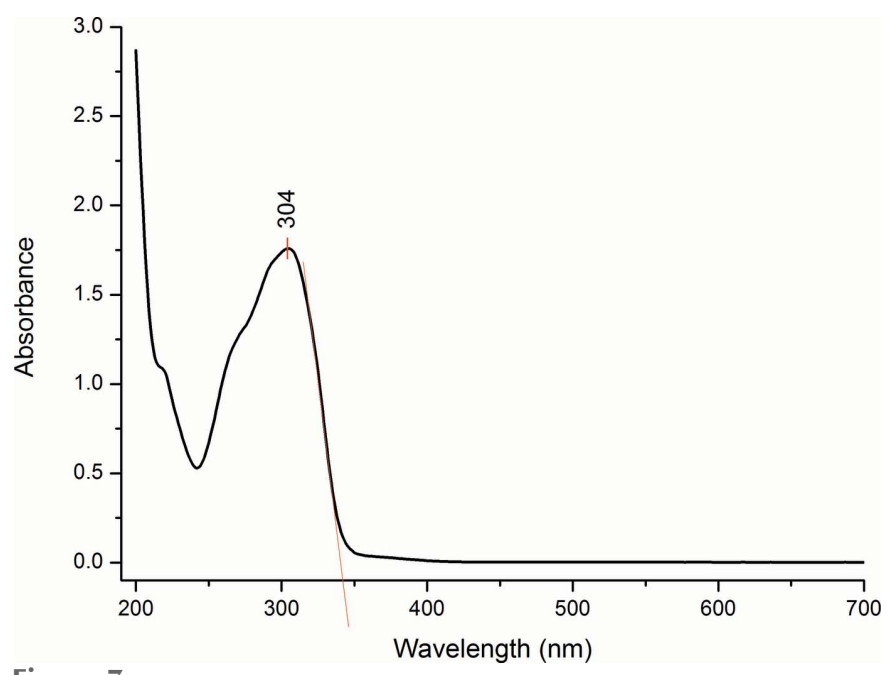

Figure 7

The UV-vis absorption spectrum of the title compound.

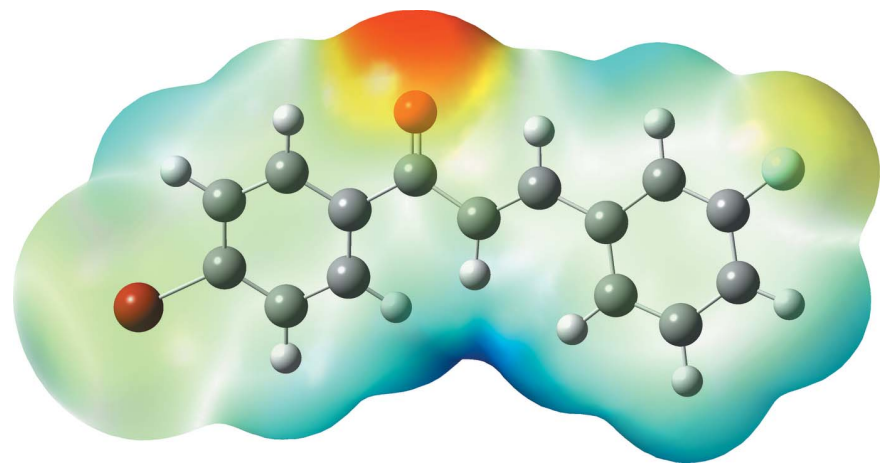

Figure 8

The molecular electrostatic potential surface of the title compound calculated at the DFT/B3LYP/6-311 G++(d,p) level.

plots of the HOMO and LUMO for the title compound were calculated using density functional theory (DFT) at the B3LYP/6-311 G++(d,p) level. As seen from the orbital plots (Fig. 6), both HOMO and LUMO extend mainly over the entire molecule, but the molecular orbital localization differs. This can be seen specifically at the enone moiety where the orbital accumulates around the carbon-carbon double bond at the HOMO state whereas it is localized at the carbon-carbon single bond at the LUMO state, indicating conjugation within the molecule. The calculated energy gap, $E_{\mathrm{LumO}}-E_{\mathrm{HOMO}}$, is $4.12 \mathrm{eV}$. The experimental UV-vis absorption spectrum consists of one major band (Fig. 7) occurring in the visible region at $304 \mathrm{~nm}$ which was assigned to the $\pi-\pi^{*}$ transition. This sharp peak was expected to arise from the carbonyl group of the chalcone (Zainuri et al., 2018). From the UV-vis absorption edge, the calculated energy band-gap value is $3.60 \mathrm{eV}$, which is similar to that found in a previous study of a related chalcone (Zaini et al., 2018).

\section{Molecular electrostatic potential}

The molecular electrostatic potential (MEP) is useful in depicting the molecular size and shape as well as in visualizing the charge distributions of molecules. The MEP map (Fig. 8) of the title compounds was calculated theoretically at the DFT/B3LYP/6-311 G++(d,p) level of theory. The colour grading in the plot represents the electrostatic potential regions in which the red-coloured region is nucleophile and electron rich, the blue colour indicates the electron-poor electrophile region and the white region indicates neutral atoms. These sites provide information about where the intermolecular interactions are involved within the molecule (Gunasekaran et al., 2008). The reactive sites are found near the carbonyl group: the region is represented in red and possesses the most negative potential spots. This nucleophile site (negative potential value of -0.04713 a.u.) is distributed around the oxygen atom due to the intermolecular $\mathrm{C}-\mathrm{H} \cdots \mathrm{O}$ interactions; in the molecular structure it indicates the strongest repulsion site (electrophilic attack), whereas the strongest attraction regions (nucleophilic attack) portrayed by the blue spots are localized on the hydrogen atoms. 
Table 2

Experimental details.

\begin{tabular}{|c|c|}
\hline \multicolumn{2}{|l|}{ Crystal data } \\
\hline Chemical formula & $\mathrm{C}_{15} \mathrm{H}_{10} \mathrm{BrFO}$ \\
\hline$M_{\mathrm{r}}$ & 305.14 \\
\hline Crystal system, space group & Triclinic, $P \overline{1}$ \\
\hline Temperature (K) & 296 \\
\hline$a, b, c(\AA)$ & $\begin{array}{l}6.0090(4), 10.8695(7) \\
20.5616(12)\end{array}$ \\
\hline$\alpha, \beta, \gamma\left({ }^{\circ}\right)$ & $102.803(1), 96.938(1), 97.276(1)$ \\
\hline$V\left(\AA^{3}\right)$ & $1283.57(14)$ \\
\hline$Z$ & 4 \\
\hline Radiation type & Мо $K \alpha$ \\
\hline$\mu\left(\mathrm{mm}^{-1}\right)$ & 3.20 \\
\hline Crystal size $(\mathrm{mm})$ & $0.56 \times 0.39 \times 0.29$ \\
\hline \multicolumn{2}{|l|}{ Data collection } \\
\hline Diffractometer & $\begin{array}{l}\text { Bruker SMART APEXII Duo } \\
\text { CCD area-detector }\end{array}$ \\
\hline Absorption correction & $\begin{array}{l}\text { Multi-scan (SADABS; Bruker, } \\
\text { 2009) }\end{array}$ \\
\hline$T_{\min }, T_{\max }$ & $0.267,0.455$ \\
\hline $\begin{array}{l}\text { No. of measured, independent and } \\
\text { observed }[I>2 \sigma(I)] \text { reflections }\end{array}$ & $27389,7437,4832$ \\
\hline$R_{\text {int }}$ & 0.037 \\
\hline$(\sin \theta / \lambda)_{\max }\left(\AA^{-1}\right)$ & 0.703 \\
\hline \multicolumn{2}{|l|}{ Refinement } \\
\hline$R\left[F^{2}>2 \sigma\left(F^{2}\right)\right], w R\left(F^{2}\right), S$ & $0.048,0.158,1.04$ \\
\hline No. of reflections & 7437 \\
\hline No. of parameters & 325 \\
\hline $\mathrm{H}$-atom treatment & $\begin{array}{l}\mathrm{H} \text { atoms treated by a mixture of } \\
\text { independent and constrained } \\
\text { refinement }\end{array}$ \\
\hline$\Delta \rho_{\max }, \Delta \rho_{\min }\left(\mathrm{e} \AA^{-3}\right)$ & $0.85,-1.35$ \\
\hline
\end{tabular}

Computer programs: APEX2 and SAINT (Bruker, 2009) and SHELXTL (Sheldrick, 2008) and PLATON (Spek, 2009).

\section{Database survey}

A search of the Cambridge Structural Database (Version 5.39, last update November 2017; Groom et al., 2016) revealed one closely related compound that differs in the halogen substitution attached to the aldehyde ring, namely 3-(3-bromophenyl)-1-(4-bromophenyl)-prop-2-en-1-one (Teh et al., 2006). Other related compounds, which differ in the halogen substitution at the para-position of the aldehyde ring include (2E)-1(4-bromophenyl)-3-(4-fluorophenyl)prop-2-en-1-one (Dutkiewicz et al., 2010), 1-(4-bromophenyl)-3-(4-chlorophenyl)prop-2-en-1-one (Yang et al., 2006), 1,3-bis(4-bromophenyl)prop-2-en-1-one (Ng et al., 2006), (E)-1-(4-bromophenyl)-3(4-iodoophenyl)prop-2-en-1-one (Zainuri et al., 2017) and (E)-3-(4-bromophenyl)-1-(4-fluorophenyl)prop-2-en-1-one (Zaini et al., 2018).

\section{Synthesis and crystallization}

The title compound was prepared by a standard ClaisenSchmidt condensation reaction at room temperature. A mixture of 4-bromoacetophenone $(0.5 \mathrm{mmol})$ and 3-fluorobenzaldehyde $(0.5 \mathrm{mmol})$ was dissolved in methanol $(20 \mathrm{ml})$ and the solution stirred continuously. A catalytic amount of $\mathrm{NaOH}(5 \mathrm{ml}, 20 \%)$ was added to the solution dropwise until a precipitate formed and the reaction was stirred continuously for about $5 \mathrm{~h}$. After stirring, the solution was poured into $60 \mathrm{ml}$ of ice-cold distilled water. The resultant crude product was filtered and washed successively with distilled water until the filtrate turned colourless. The dried precipitate was further recrystallized to obtain the desired chalcone. Crystals suitable for X-ray diffraction analysis were formed by slow evaporation of an acetone solution.

\section{Refinement}

Details of the crystal data collection and structure refinement are summarized in Table 2. All C-bound $\mathrm{H}$ atoms were positioned geometrically $(\mathrm{C}-\mathrm{H}=0.930 \AA$ ) and refined using a riding model with $U_{\text {iso }}(\mathrm{H})=1.2 U_{\text {eq }}(\mathrm{C})$. One outlier (3i11) was omitted in the last cycles of refinement.

\section{Funding information}

The authors would like to thank the Malaysian Government and Universiti Sains Malaysia (USM) for providing facilities and the Fundamental Research Grant Scheme (FRGS) No. 203.PFIZIK.6711606 for supplying the chemicals to conduct this research successfully.

\section{References}

Bruker (2009). APEX2, SAINT and SADABS. Bruker AXS Inc., Madison, Wisconsin, USA.

Di Carlo, G., Mascolo, N., Izzo, A. A. \& Capasso, F. (1999). Life Sci. 65, 337-353.

Dutkiewicz, G., Veena, K., Narayana, B., Yathirajan, H. S. \& Kubicki, M. (2010). Acta Cryst. E66, o1243-01244.

Frisch, M. J., Trucks, G. W., Schlegel, H. B., Scuseria, G. E., Robb, M. A., Cheeseman, J. R., Scalmani, G., Barone, V., Mennucci, B., Petersson, G. A., Nakatsuji, H., Caricato, M., Li, X., Hratchian, H. P., Izmaylov, A. F., Bloino, J., Zheng, G., Sonnenberg, J. L., Hada, M., Ehara, M., Toyota, K., Fukuda, R., Hasegawa, J., Ishida, M., Nakajima, T., Honda, Y., Kitao, O., Nakai, H., Vreven, T., Montgomery, J. A. Jr, Peralta, J. E., Ogliaro, F., Bearpark, M., Heyd, J. J., Brothers, E., Kudin, K. N., Staroverov, V. N., Keith, T., Kobayashi, R., Normand, J., Raghavachari, K., Rendell, A., Burant, J. C., Iyengar, S. S., Tomasi, J., Cossi, M., Rega, N., Millam, J. M., Klene, M., Knox, J. E., Cross, J. B., Bakken, V., Adamo, C., Jaramillo, J., Gomperts, R., Stratmann, R. E., Yazyev, O., Austin, A. J., Cammi, R., Pomelli, C., Ochterski, J. W., Martin, R. L., Morokuma, K., Zakrzewski, V. G., Voth, G. A., Salvador, P., Dannenberg, J. J., Dapprich, S., Daniels, A. D., Farkas, O., Foresman, J. B., Ortiz, J. V., Cioslowski, J. \& Fox, D. J. (2009). GAUSSIAN09. Gaussian Inc., USA.

Goto, Y., Hayashi, A., Kimura, Y. \& Nakayama, M. (1991). J. Cryst. Growth, 108, 688-698.

Groom, C. R., Bruno, I. J., Lightfoot, M. P. \& Ward, S. C. (2016). Acta Cryst. B72, 171-179.

Gunasekaran, S., Kumaresan, S., Arunbalaji, R., Anand, G. \& Srinivasan, S. (2008). J. Chem. Sci. 120, 780-785.

Ng, S.-L., Shettigar, V., Razak, I. A., Fun, H.-K., Patil, P. S. \& Dharmaprakash, S. M. (2006). Acta Cryst. E62, o1421-o1423.

O'Hagan, D. (2008). Chem. Soc. Rev. 37, 308-319.

Patil, P. S., Teh, J. B.-J., Fun, H.-K., Razak, I. A. \& Dharmaprakash, S. M. (2006a). Acta Cryst. E62, o896-o898.

Patil, P. S., Teh, J. B.-J., Fun, H.-K., Razak, I. A. \& Dharmaprakash, S. M. (2006b). Acta Cryst. E62, o1710-o1712.

Sheldrick, G. M. (2008). Acta Cryst. A64, 112-122. 
Spek, A. L. (2009). Acta Cryst. D65, 148-155.

Teh, J. B.-J., Patil, P. S., Fun, H.-K., Razak, I. A. \& Dharmaprakash, S. M. (2006). Acta Cryst. E62, o2399-o2400.

Ternavisk, R. R., Camargo, A. J., Machado, F. B. C., Rocco, J. A. F. F., Aquino, G. L. B., Silva, V. H. C. \& Napolitano, H. B. (2014). J. Mol. Model. 20, 2526-2536.

Thanigaimani, K., Arshad, S., Khalib, N. C., Razak, I. A., Arunagiri, C., Subashini, A., Sulaiman, S. F., Hashim, N. S. \& Ooi, K. L. (2015). Spectrochim. Acta A Mol. Biomol. Spectrosc. 149, 90-102.

Wolff, S. K., Grimwood, D. J., McKinnon, J. J., Turner, M. J., Jayatilaka, D. \& Spackman, M. A. (2012). CrystalExplorer. University of Western Australia, Perth.
Yang, W., Wang, L. \& Zhang, D. (2006). J. Chem. Crystallogr. 36, 195198.

Zaini, M. F., Arshad, S., Ibrahim, A. R., Khalib, N. C. \& Zainuri, D. A. (2018). J. Phys. Conf. Ser. 1083, 012047.

Zainuri, D. A., Arshad, S., Khalib, N. C., Razak, I. A., Pillai, R. R., Sulaiman, S. F., Hashim, N. S., Ooi, K. L., Armaković, S., Armaković, S. J., Panicker, C. Y. \& Van Alsenoy, C. (2017). J. Mol. Struct. 1128, 520-533.

Zainuri, D. A., Razak, I. A. \& Arshad, S. (2018). Acta Cryst. E74, 1427-1432.

Zhao, B., Lu, W.-Q., Zhou, Z.-H. \& Wu, Y. (2000). J. Mater. Chem. 10, 1513-1517. 


\section{supporting information}

Acta Cryst. (2019). E75, 58-63 [https://doi.org/10.1107/S2056989018017371]

Crystal structure, Hirshfeld surface analysis and DFT studies of (E)-1-(4-bromophenyl)-3-(3-fluorophenyl)prop-2-en-1-one

Muhamad Fikri Zaini, Ibrahim Abdul Razak, Mohamad Zahid Anis and Suhana Arshad

Computing details

Data collection: APEX2 (Bruker, 2009); cell refinement: SAINT (Bruker, 2009); data reduction: SAINT (Bruker, 2009); program(s) used to solve structure: SHELXTL (Sheldrick, 2008); program(s) used to refine structure: SHELXTL

(Sheldrick, 2008); molecular graphics: SHELXTL (Sheldrick, 2008); software used to prepare material for publication:

SHELXTL (Sheldrick, 2008) and PLATON (Spek, 2009).

(E)-1-(4-Bromophenyl)-3-(3-fluorophenyl)prop-2-en-1-one

Crystal data

$\mathrm{C}_{15} \mathrm{H}_{10} \mathrm{BrFO}$

$M_{r}=305.14$

Triclinic, $P \overline{1}$

Hall symbol: -P 1

$a=6.0090(4) \AA$

$b=10.8695(7) \AA$

$c=20.5616(12) \AA$

$\alpha=102.803(1)^{\circ}$

$\beta=96.938(1)^{\circ}$

$\gamma=97.276(1)^{\circ}$

$V=1283.57(14) \AA^{3}$

\section{Data collection}

Bruker SMART APEXII Duo CCD areadetector diffractometer

Radiation source: fine-focus sealed tube Graphite monochromator $\varphi$ and $\omega$ scans

Absorption correction: multi-scan (SADABS; Bruker, 2009)

$T_{\min }=0.267, T_{\max }=0.455$

Refinement

Refinement on $F^{2}$

Least-squares matrix: full

$R\left[F^{2}>2 \sigma\left(F^{2}\right)\right]=0.048$

$w R\left(F^{2}\right)=0.158$

$S=1.04$

7437 reflections

325 parameters

\section{$Z=4$}

$F(000)=608$

$D_{\mathrm{x}}=1.579 \mathrm{Mg} \mathrm{m}^{-3}$

Mo $K \alpha$ radiation, $\lambda=0.71073 \AA$

Cell parameters from 8958 reflections

$\theta=2.5-28.0^{\circ}$

$\mu=3.20 \mathrm{~mm}^{-1}$

$T=296 \mathrm{~K}$

Plate, yellow

$0.56 \times 0.39 \times 0.29 \mathrm{~mm}$

27389 measured reflections

7437 independent reflections

4832 reflections with $I>2 \sigma(I)$

$R_{\text {int }}=0.037$

$\theta_{\text {max }}=30.0^{\circ}, \theta_{\min }=2.0^{\circ}$

$h=-8 \rightarrow 8$

$k=-15 \rightarrow 15$

$l=-28 \rightarrow 28$

0 restraints

Primary atom site location: structure-invariant direct methods

Secondary atom site location: difference Fourier map

Hydrogen site location: inferred from neighbouring sites 
$\mathrm{H}$ atoms treated by a mixture of independent and constrained refinement

$w=1 /\left[\sigma^{2}\left(F_{\mathrm{o}}^{2}\right)+(0.0792 P)^{2}+0.4733 P\right]$

where $P=\left(F_{\mathrm{o}}^{2}+2 F_{\mathrm{c}}^{2}\right) / 3$

$$
\begin{aligned}
& (\Delta / \sigma)_{\max }=0.001 \\
& \Delta \rho_{\max }=0.85 \mathrm{e} \AA^{-3} \\
& \Delta \rho_{\min }=-1.35 \mathrm{e} \AA^{-3}
\end{aligned}
$$

\section{Special details}

Geometry. All esds (except the esd in the dihedral angle between two 1.s. planes) are estimated using the full covariance matrix. The cell esds are taken into account individually in the estimation of esds in distances, angles and torsion angles; correlations between esds in cell parameters are only used when they are defined by crystal symmetry. An approximate (isotropic) treatment of cell esds is used for estimating esds involving l.s. planes.

Refinement. Refinement of $\mathrm{F}^{2}$ against ALL reflections. The weighted R-factor wR and goodness of fit $\mathrm{S}$ are based on $\mathrm{F}^{2}$, conventional R-factors $\mathrm{R}$ are based on $\mathrm{F}$, with $\mathrm{F}$ set to zero for negative $\mathrm{F}^{2}$. The threshold expression of $\mathrm{F}^{2}>2$ sigma $\left(\mathrm{F}^{2}\right)$ is used only for calculating R-factors ( $\mathrm{gt}$ ) etc. and is not relevant to the choice of reflections for refinement. R-factors based

\begin{tabular}{|c|c|c|c|c|}
\hline & $x$ & $y$ & $z$ & $U_{\text {iso }} * / U_{\text {eq }}$ \\
\hline Br1A & $-0.36851(7)$ & $1.01488(4)$ & 0.369778 (19) & $0.07958(15)$ \\
\hline F1A & $0.5304(4)$ & $0.3263(2)$ & $0.74576(11)$ & $0.0894(7)$ \\
\hline $\mathrm{O} 1 \mathrm{~A}$ & $0.3367(4)$ & $0.6239(3)$ & $0.46486(13)$ & $0.0805(8)$ \\
\hline $\mathrm{C} 1 \mathrm{~A}$ & $-0.1316(5)$ & $0.7958(3)$ & $0.49478(14)$ & $0.0533(6)$ \\
\hline H1AA & -0.1703 & 0.7725 & 0.5333 & $0.064 *$ \\
\hline $\mathrm{C} 2 \mathrm{~A}$ & $-0.2576(5)$ & $0.8732(3)$ & $0.46588(16)$ & $0.0595(7)$ \\
\hline H2AA & -0.3807 & 0.9017 & 0.4846 & $0.071 *$ \\
\hline $\mathrm{C} 3 \mathrm{~A}$ & $-0.1989(5)$ & $0.9074(3)$ & $0.40938(15)$ & $0.0564(7)$ \\
\hline $\mathrm{C} 4 \mathrm{~A}$ & $-0.0175(6)$ & $0.8661(3)$ & $0.38068(16)$ & $0.0632(8)$ \\
\hline H4AA & 0.0205 & 0.8903 & 0.3423 & $0.076^{*}$ \\
\hline C5A & $0.1071(5)$ & $0.7882(3)$ & $0.40975(15)$ & $0.0583(7)$ \\
\hline H5AA & 0.2293 & 0.7596 & 0.3906 & $0.070^{*}$ \\
\hline C6A & $0.0523(4)$ & $0.7524(2)$ & $0.46713(13)$ & $0.0462(5)$ \\
\hline C7A & $0.1930(5)$ & $0.6668(3)$ & $0.49525(14)$ & $0.0526(6)$ \\
\hline C8A & $0.1559(5)$ & $0.6354(3)$ & $0.55997(14)$ & $0.0513(6)$ \\
\hline H8AA & 0.0430 & 0.6682 & 0.5824 & $0.062 *$ \\
\hline C9A & $0.2801(5)$ & $0.5616(3)$ & $0.58648(13)$ & $0.0492(6)$ \\
\hline H9AA & 0.3932 & 0.5324 & 0.5628 & $0.059 *$ \\
\hline $\mathrm{C} 10 \mathrm{~A}$ & $0.2592(5)$ & $0.5208(2)$ & $0.64885(12)$ & $0.0457(5)$ \\
\hline C11A & $0.4081(5)$ & 0.4430 & $0.66883(14)$ & $0.0531(6)$ \\
\hline H11A & 0.5195 & 0.4181 & 0.6433 & $0.064 *$ \\
\hline $\mathrm{C} 12 \mathrm{~A}$ & $0.3866(6)$ & $0.4042(3)$ & $0.72699(15)$ & $0.0606(7)$ \\
\hline C13A & $0.2265(6)$ & 0.4381 & $0.76688(15)$ & $0.0685(9)$ \\
\hline H13A & 0.2171 & 0.4102 & 0.8062 & $0.082 *$ \\
\hline C14A & $0.0807(7)$ & $0.5147(4)$ & $0.74667(17)$ & $0.0750(9)$ \\
\hline H14A & -0.0297 & 0.5390 & 0.7727 & $0.090 *$ \\
\hline C15A & $0.0953(6)$ & $0.5562(3)$ & $0.68819(15)$ & $0.0637(8)$ \\
\hline $\mathrm{H} 15 \mathrm{~A}$ & -0.0050 & 0.6080 & 0.6752 & $0.076^{*}$ \\
\hline Br1B & $-0.33082(7)$ & $-0.20429(4)$ & $0.72238(2)$ & $0.09061(17)$ \\
\hline F1B & $1.2882(4)$ & 0.0939 (2) & $1.06936(14)$ & $0.1021(8)$ \\
\hline O1B & $0.3006(4)$ & 0.34757 (19) & $0.91642(11)$ & $0.0632(5)$ \\
\hline
\end{tabular}
on $\mathrm{F}^{2}$ are statistically about twice as large as those based on F, and R-factors based on ALL data will be even larger.

Fractional atomic coordinates and isotropic or equivalent isotropic displacement parameters $\left(\AA^{2}\right)$ 


$\begin{array}{lllll}\text { C1B } & -0.0157(5) & 0.1510(3) & 0.83454(15) & 0.0538(6) \\ \text { H1BA } & -0.0513 & 0.2333 & 0.8440 & 0.065^{*} \\ \text { C2B } & -0.1694(5) & 0.0535(3) & 0.79170(15) & 0.0587(7) \\ \text { H2BA } & -0.3074 & 0.0694 & 0.7722 & 0.070^{*} \\ \text { C3B } & -0.1153(5) & -0.0677(3) & 0.77835(16) & 0.0590(7) \\ \text { C4B } & 0.0883(6) & -0.0930(3) & 0.80586(19) & 0.0730(9) \\ \text { H4BA } & 0.1231 & -0.1754 & 0.7959 & 0.088^{*} \\ \text { C5B } & 0.2415(5) & 0.0063(3) & 0.84882(17) & 0.0646(8) \\ \text { H5BA } & 0.3799 & -0.0101 & 0.8677 & 0.077^{*} \\ \text { C6B } & 0.1921(5) & 0.1289(3) & 0.86397(13) & 0.0484(6) \\ \text { C7B } & 0.3501(5) & 0.2405(2) & 0.90990(13) & 0.0483(6) \\ \text { C8B } & 0.5623(5) & 0.2192(3) & 0.94716(14) & 0.0522(6) \\ \text { H8BA } & 0.6019 & 0.1378 & 0.9396 & 0.063^{*} \\ \text { C9B } & 0.6961(5) & 0.3151(3) & 0.99126(13) & 0.0481(5) \\ \text { H9BA } & 0.6479 & 0.3943 & 0.9971 & 0.058^{*} \\ \text { C10B } & 0.9115(5) & 0.3113(2) & 1.03206(12) & 0.0458(5) \\ \text { C11B } & 0.9974(5) & 0.1983(3) & 1.03227(15) & 0.0574(7) \\ \text { H11B } & 0.9164 & 0.1202 & 1.0071 & 0.069^{*} \\ \text { C12B } & 1.2032(6) & 0.2045(3) & 1.07027(16) & 0.0623(7) \\ \text { C13B } & 1.3312(5) & 0.3157(3) & 1.10868(15) & 0.0611(7) \\ \text { H13B } & 1.4703 & 0.3159 & 1.1341 & 0.073^{*} \\ \text { C14B } & 1.2459(6) & 0.4271(3) & 1.10829(14) & 0.0607(7) \\ \text { H14B } & 1.3291 & 0.5046 & 1.1335 & 0.073^{*} \\ \text { C15B } & 1.0377(5) & 0.4254(3) & 1.07085(13) & 0.0527(6) \\ \text { H15B } & 0.9816 & 0.5016 & 1.0717 & 0.063^{*}\end{array}$

Atomic displacement parameters $\left(\AA^{2}\right)$

\begin{tabular}{lllllll}
\hline & $U^{11}$ & $U^{22}$ & $U^{33}$ & $U^{12}$ & $U^{13}$ & $U^{23}$ \\
\hline Br1A & $0.0856(3)$ & $0.0670(2)$ & $0.0818(3)$ & $0.01918(18)$ & $-0.02313(19)$ & $0.02390(17)$ \\
F1A & $0.1164(18)$ & $0.0866(14)$ & $0.0753(13)$ & $0.0378(13)$ & $-0.0027(12)$ & $0.0375(11)$ \\
O1A & $0.0840(17)$ & $0.1058(19)$ & $0.0857(16)$ & $0.0548(15)$ & $0.0438(14)$ & $0.0557(15)$ \\
C1A & $0.0548(15)$ & $0.0585(16)$ & $0.0497(14)$ & $0.0147(12)$ & $0.0070(12)$ & $0.0166(12)$ \\
C2A & $0.0569(16)$ & $0.0606(17)$ & $0.0612(16)$ & $0.0184(13)$ & $0.0026(13)$ & $0.0134(13)$ \\
C3A & $0.0599(16)$ & $0.0471(14)$ & $0.0552(15)$ & $0.0058(12)$ & $-0.0141(13)$ & $0.0115(11)$ \\
C4A & $0.073(2)$ & $0.0651(18)$ & $0.0542(16)$ & $0.0068(15)$ & $0.0018(14)$ & $0.0268(14)$ \\
C5A & $0.0599(17)$ & $0.0622(17)$ & $0.0578(16)$ & $0.0161(13)$ & $0.0103(13)$ & $0.0206(13)$ \\
C6A & $0.0467(13)$ & $0.0440(12)$ & $0.0471(13)$ & $0.0050(10)$ & $0.0029(10)$ & $0.0128(10)$ \\
C7A & $0.0535(15)$ & $0.0521(14)$ & $0.0570(15)$ & $0.0134(12)$ & $0.0103(12)$ & $0.0191(12)$ \\
C8A & $0.0537(15)$ & $0.0519(14)$ & $0.0521(14)$ & $0.0143(12)$ & $0.0119(12)$ & $0.0153(11)$ \\
C9A & $0.0504(14)$ & $0.0487(13)$ & $0.0495(13)$ & $0.0093(11)$ & $0.0076(11)$ & $0.0129(11)$ \\
C10A & $0.0509(14)$ & $0.0421(12)$ & $0.0417(12)$ & $0.0054(10)$ & $0.0032(10)$ & $0.0083(10)$ \\
C11A & $0.0605(16)$ & $0.0479(13)$ & $0.0482(14)$ & $0.0101(12)$ & $0.0009(12)$ & $0.0090(11)$ \\
C12A & $0.075(2)$ & $0.0516(15)$ & $0.0514(15)$ & $0.0051(13)$ & $-0.0077(14)$ & $0.0159(12)$ \\
C13A & $0.083(2)$ & $0.078(2)$ & $0.0440(15)$ & $0.0016(17)$ & $0.0046(15)$ & $0.0221(14)$ \\
C14A & $0.077(2)$ & $0.098(3)$ & $0.0557(17)$ & $0.021(2)$ & $0.0217(16)$ & $0.0213(18)$ \\
C15A & $0.071(2)$ & $0.0728(19)$ & $0.0533(16)$ & $0.0248(16)$ & $0.0120(14)$ & $0.0185(14)$ \\
Br1B & $0.0727(3)$ & $0.0615(2)$ & $0.1139(3)$ & $0.01018(17)$ & $-0.0328(2)$ & $-0.00307(19)$
\end{tabular}




\begin{tabular}{lllllll}
\hline & & & & & \\
F1B & $0.0907(16)$ & $0.0653(13)$ & $0.140(2)$ & $0.0298(11)$ & $-0.0343(15)$ & $0.0209(13)$ \\
O1B & $0.0684(13)$ & $0.0461(10)$ & $0.0725(13)$ & $0.0170(9)$ & $-0.0053(10)$ & $0.0128(9)$ \\
C1B & $0.0542(16)$ & $0.0488(13)$ & $0.0599(15)$ & $0.0171(11)$ & $0.0020(12)$ & $0.0147(12)$ \\
C2B & $0.0498(15)$ & $0.0613(17)$ & $0.0632(17)$ & $0.0151(13)$ & $-0.0054(13)$ & $0.0153(13)$ \\
C3B & $0.0537(16)$ & $0.0529(15)$ & $0.0638(16)$ & $0.0070(12)$ & $-0.0068(13)$ & $0.0091(13)$ \\
C4B & $0.0655(19)$ & $0.0492(16)$ & $0.093(2)$ & $0.0170(14)$ & $-0.0145(17)$ & $0.0023(15)$ \\
C5B & $0.0523(16)$ & $0.0541(16)$ & $0.081(2)$ & $0.0168(13)$ & $-0.0117(14)$ & $0.0102(14)$ \\
C6B & $0.0473(13)$ & $0.0519(14)$ & $0.0472(13)$ & $0.0113(11)$ & $0.0024(11)$ & $0.0151(11)$ \\
C7B & $0.0513(14)$ & $0.0462(13)$ & $0.0483(13)$ & $0.0105(11)$ & $0.0034(11)$ & $0.0138(10)$ \\
C8B & $0.0528(15)$ & $0.0472(13)$ & $0.0567(15)$ & $0.0134(11)$ & $0.0017(12)$ & $0.0134(11)$ \\
C9B & $0.0520(14)$ & $0.0462(13)$ & $0.0477(13)$ & $0.0114(11)$ & $0.0055(11)$ & $0.0135(10)$ \\
C10B & $0.0506(14)$ & $0.0462(13)$ & $0.0410(12)$ & $0.0097(10)$ & $0.0056(10)$ & $0.0112(10)$ \\
C11B & $0.0600(16)$ & $0.0450(13)$ & $0.0626(16)$ & $0.0068(12)$ & $-0.0056(13)$ & $0.0125(12)$ \\
C12B & $0.0648(18)$ & $0.0560(16)$ & $0.0659(17)$ & $0.0164(14)$ & $-0.0031(14)$ & $0.0176(14)$ \\
C13B & $0.0552(16)$ & $0.0719(19)$ & $0.0547(15)$ & $0.0077(14)$ & $-0.0022(13)$ & $0.0190(14)$ \\
C14B & $0.0633(18)$ & $0.0603(17)$ & $0.0484(14)$ & $0.0039(13)$ & $-0.0039(13)$ & $0.0017(12)$ \\
C15B & $0.0635(17)$ & $0.0485(14)$ & $0.0438(13)$ & $0.0121(12)$ & $0.0054(12)$ & $0.0060(11)$ \\
& & & & & \\
\hline
\end{tabular}

Geometric parameters $\left(\AA,{ }^{\circ}\right)$

\begin{tabular}{llll}
\hline Br1A-C3A & $1.894(3)$ & Br1B-C3B & $1.898(3)$ \\
F1A-C12A & $1.365(4)$ & F1B-C12B & $1.362(4)$ \\
O1A-C7A & $1.207(4)$ & O1B-C7B & $1.221(3)$ \\
C1A-C2A & $1.381(4)$ & C1B-C2B & $1.376(4)$ \\
C1A-C6A & $1.390(4)$ & C1B-C6B & $1.393(4)$ \\
C1A-H1AA & 0.9300 & C1B-H1BA & 0.9300 \\
C2A-C3A & $1.367(5)$ & C2B-C3B & $1.374(4)$ \\
C2A-H2AA & 0.9300 & C2B-H2BA & 0.9300 \\
C3A-C4A & $1.378(5)$ & C3B-C4B & $1.371(4)$ \\
C4A-C5A & $1.383(4)$ & C4B-C5B & $1.386(4)$ \\
C4A-H4AA & 0.9300 & C4B-H4BA & 0.9300 \\
C5A-C6A & $1.385(4)$ & C5B-C6B & $1.379(4)$ \\
C5A-H5AA & 0.9300 & C5B-H5BA & 0.9300 \\
C6A-C7A & $1.494(4)$ & C6B-C7B & $1.499(4)$ \\
C7A-C8A & $1.481(4)$ & C7B-C8B & $1.477(4)$ \\
C8A-C9A & $1.321(4)$ & C8B-C9B & $1.322(4)$ \\
C8A-H8AA & 0.9300 & C8B-H8BA & 0.9300 \\
C9A-C10A & $1.461(4)$ & C9B-C10B & $1.465(4)$ \\
C9A-H9AA & 0.9300 & C9B-H9BA & 0.9300 \\
C10A-C15A & $1.387(4)$ & C10B-C15B & $1.388(4)$ \\
C10A-C11A & $1.393(4)$ & C10B-C11B & $1.393(4)$ \\
C11A-C12A & $1.368(4)$ & C11B-C12B & $1.366(4)$ \\
C11A-H11A & 0.9300 & C11B-H11B & 0.9300 \\
C12A-C13A & $1.371(5)$ & C12B-C13B & $1.368(5)$ \\
C13A-C14A & $1.373(5)$ & C13B-C14B & $1.375(5)$ \\
C13A-H13A & 0.9300 & C13B-H13B & 0.9300 \\
C14A-C15A & $1.383(5)$ & C14B-C15B & $1.383(4)$ \\
C14A-H14A & 0.9300 & C14B-H14B & 0.9300
\end{tabular}




\begin{tabular}{|c|c|c|c|}
\hline $\mathrm{C} 15 \mathrm{~A}-\mathrm{H} 15 \mathrm{~A}$ & 0.9300 & $\mathrm{C} 15 \mathrm{~B}-\mathrm{H} 15 \mathrm{~B}$ & 0.9300 \\
\hline $\mathrm{C} 2 \mathrm{~A}-\mathrm{C} 1 \mathrm{~A}-\mathrm{C} 6 \mathrm{~A}$ & $121.0(3)$ & $\mathrm{C} 2 \mathrm{~B}-\mathrm{C} 1 \mathrm{~B}-\mathrm{C} 6 \mathrm{~B}$ & $121.4(3)$ \\
\hline $\mathrm{C} 2 \mathrm{~A}-\mathrm{C} 1 \mathrm{~A}-\mathrm{H} 1 \mathrm{AA}$ & 119.5 & $\mathrm{C} 2 \mathrm{~B}-\mathrm{C} 1 \mathrm{~B}-\mathrm{H} 1 \mathrm{BA}$ & 119.3 \\
\hline $\mathrm{C} 6 \mathrm{~A}-\mathrm{C} 1 \mathrm{~A}-\mathrm{H} 1 \mathrm{AA}$ & 119.5 & $\mathrm{C} 6 \mathrm{~B}-\mathrm{C} 1 \mathrm{~B}-\mathrm{H} 1 \mathrm{BA}$ & 119.3 \\
\hline $\mathrm{C} 3 \mathrm{~A}-\mathrm{C} 2 \mathrm{~A}-\mathrm{C} 1 \mathrm{~A}$ & $119.1(3)$ & $\mathrm{C} 3 \mathrm{~B}-\mathrm{C} 2 \mathrm{~B}-\mathrm{C} 1 \mathrm{~B}$ & $118.8(3)$ \\
\hline $\mathrm{C} 3 \mathrm{~A}-\mathrm{C} 2 \mathrm{~A}-\mathrm{H} 2 \mathrm{AA}$ & 120.4 & $\mathrm{C} 3 \mathrm{~B}-\mathrm{C} 2 \mathrm{~B}-\mathrm{H} 2 \mathrm{BA}$ & 120.6 \\
\hline $\mathrm{C} 1 \mathrm{~A}-\mathrm{C} 2 \mathrm{~A}-\mathrm{H} 2 \mathrm{AA}$ & 120.4 & $\mathrm{C} 1 \mathrm{~B}-\mathrm{C} 2 \mathrm{~B}-\mathrm{H} 2 \mathrm{BA}$ & 120.6 \\
\hline $\mathrm{C} 2 \mathrm{~A}-\mathrm{C} 3 \mathrm{~A}-\mathrm{C} 4 \mathrm{~A}$ & $121.4(3)$ & $\mathrm{C} 4 \mathrm{~B}-\mathrm{C} 3 \mathrm{~B}-\mathrm{C} 2 \mathrm{~B}$ & $121.6(3)$ \\
\hline $\mathrm{C} 2 \mathrm{~A}-\mathrm{C} 3 \mathrm{~A}-\mathrm{Br} 1 \mathrm{~A}$ & $119.5(2)$ & $\mathrm{C} 4 \mathrm{~B}-\mathrm{C} 3 \mathrm{~B}-\mathrm{Br} 1 \mathrm{~B}$ & $119.2(2)$ \\
\hline $\mathrm{C} 4 \mathrm{~A}-\mathrm{C} 3 \mathrm{~A}-\mathrm{Br} 1 \mathrm{~A}$ & $119.0(2)$ & $\mathrm{C} 2 \mathrm{~B}-\mathrm{C} 3 \mathrm{~B}-\mathrm{Br} 1 \mathrm{~B}$ & $119.2(2)$ \\
\hline $\mathrm{C} 3 \mathrm{~A}-\mathrm{C} 4 \mathrm{~A}-\mathrm{C} 5 \mathrm{~A}$ & $119.1(3)$ & $\mathrm{C} 3 \mathrm{~B}-\mathrm{C} 4 \mathrm{~B}-\mathrm{C} 5 \mathrm{~B}$ & $118.9(3)$ \\
\hline $\mathrm{C} 3 \mathrm{~A}-\mathrm{C} 4 \mathrm{~A}-\mathrm{H} 4 \mathrm{AA}$ & 120.5 & $\mathrm{C} 3 \mathrm{~B}-\mathrm{C} 4 \mathrm{~B}-\mathrm{H} 4 \mathrm{BA}$ & 120.5 \\
\hline $\mathrm{C} 5 \mathrm{~A}-\mathrm{C} 4 \mathrm{~A}-\mathrm{H} 4 \mathrm{AA}$ & 120.5 & $\mathrm{C} 5 \mathrm{~B}-\mathrm{C} 4 \mathrm{~B}-\mathrm{H} 4 \mathrm{BA}$ & 120.5 \\
\hline $\mathrm{C} 4 \mathrm{~A}-\mathrm{C} 5 \mathrm{~A}-\mathrm{C} 6 \mathrm{~A}$ & $120.8(3)$ & $\mathrm{C} 6 \mathrm{~B}-\mathrm{C} 5 \mathrm{~B}-\mathrm{C} 4 \mathrm{~B}$ & $121.1(3)$ \\
\hline $\mathrm{C} 4 \mathrm{~A}-\mathrm{C} 5 \mathrm{~A}-\mathrm{H} 5 \mathrm{AA}$ & 119.6 & $\mathrm{C} 6 \mathrm{~B}-\mathrm{C} 5 \mathrm{~B}-\mathrm{H} 5 \mathrm{BA}$ & 119.5 \\
\hline $\mathrm{C} 6 \mathrm{~A}-\mathrm{C} 5 \mathrm{~A}-\mathrm{H} 5 \mathrm{AA}$ & 119.6 & $\mathrm{C} 4 \mathrm{~B}-\mathrm{C} 5 \mathrm{~B}-\mathrm{H} 5 \mathrm{BA}$ & 119.5 \\
\hline $\mathrm{C} 5 \mathrm{~A}-\mathrm{C} 6 \mathrm{~A}-\mathrm{C} 1 \mathrm{~A}$ & $118.6(3)$ & $\mathrm{C} 5 \mathrm{~B}-\mathrm{C} 6 \mathrm{~B}-\mathrm{C} 1 \mathrm{~B}$ & $118.2(3)$ \\
\hline $\mathrm{C} 5 \mathrm{~A}-\mathrm{C} 6 \mathrm{~A}-\mathrm{C} 7 \mathrm{~A}$ & $117.8(2)$ & $\mathrm{C} 5 \mathrm{~B}-\mathrm{C} 6 \mathrm{~B}-\mathrm{C} 7 \mathrm{~B}$ & $123.8(2)$ \\
\hline $\mathrm{C} 1 \mathrm{~A}-\mathrm{C} 6 \mathrm{~A}-\mathrm{C} 7 \mathrm{~A}$ & $123.6(2)$ & $\mathrm{C} 1 \mathrm{~B}-\mathrm{C} 6 \mathrm{~B}-\mathrm{C} 7 \mathrm{~B}$ & $118.0(2)$ \\
\hline $\mathrm{O} 1 \mathrm{~A}-\mathrm{C} 7 \mathrm{~A}-\mathrm{C} 8 \mathrm{~A}$ & $120.4(3)$ & $\mathrm{O} 1 \mathrm{~B}-\mathrm{C} 7 \mathrm{~B}-\mathrm{C} 8 \mathrm{~B}$ & $120.9(3)$ \\
\hline $\mathrm{O} 1 \mathrm{~A}-\mathrm{C} 7 \mathrm{~A}-\mathrm{C} 6 \mathrm{~A}$ & $119.5(2)$ & $\mathrm{O} 1 \mathrm{~B}-\mathrm{C} 7 \mathrm{~B}-\mathrm{C} 6 \mathrm{~B}$ & $119.5(2)$ \\
\hline $\mathrm{C} 8 \mathrm{~A}-\mathrm{C} 7 \mathrm{~A}-\mathrm{C} 6 \mathrm{~A}$ & $120.0(2)$ & $\mathrm{C} 8 \mathrm{~B}-\mathrm{C} 7 \mathrm{~B}-\mathrm{C} 6 \mathrm{~B}$ & $119.6(2)$ \\
\hline $\mathrm{C} 9 \mathrm{~A}-\mathrm{C} 8 \mathrm{~A}-\mathrm{C} 7 \mathrm{~A}$ & $121.3(3)$ & $\mathrm{C} 9 \mathrm{~B}-\mathrm{C} 8 \mathrm{~B}-\mathrm{C} 7 \mathrm{~B}$ & $120.4(2)$ \\
\hline C9A-C8A-H8AA & 119.4 & $\mathrm{C} 9 \mathrm{~B}-\mathrm{C} 8 \mathrm{~B}-\mathrm{H} 8 \mathrm{BA}$ & 119.8 \\
\hline $\mathrm{C} 7 \mathrm{~A}-\mathrm{C} 8 \mathrm{~A}-\mathrm{H} 8 \mathrm{AA}$ & 119.4 & $\mathrm{C} 7 \mathrm{~B}-\mathrm{C} 8 \mathrm{~B}-\mathrm{H} 8 \mathrm{BA}$ & 119.8 \\
\hline $\mathrm{C} 8 \mathrm{~A}-\mathrm{C} 9 \mathrm{~A}-\mathrm{C} 10 \mathrm{~A}$ & $127.1(3)$ & $\mathrm{C} 8 \mathrm{~B}-\mathrm{C} 9 \mathrm{~B}-\mathrm{C} 10 \mathrm{~B}$ & $127.6(2)$ \\
\hline $\mathrm{C} 8 \mathrm{~A}-\mathrm{C} 9 \mathrm{~A}-\mathrm{H} 9 \mathrm{AA}$ & 116.4 & $\mathrm{C} 8 \mathrm{~B}-\mathrm{C} 9 \mathrm{~B}-\mathrm{H} 9 \mathrm{BA}$ & 116.2 \\
\hline $\mathrm{C} 10 \mathrm{~A}-\mathrm{C} 9 \mathrm{~A}-\mathrm{H} 9 \mathrm{AA}$ & 116.4 & $\mathrm{C} 10 \mathrm{~B}-\mathrm{C} 9 \mathrm{~B}-\mathrm{H} 9 \mathrm{BA}$ & 116.2 \\
\hline $\mathrm{C} 15 \mathrm{~A}-\mathrm{C} 10 \mathrm{~A}-\mathrm{C} 11 \mathrm{~A}$ & $119.3(2)$ & $\mathrm{C} 15 \mathrm{~B}-\mathrm{C} 10 \mathrm{~B}-\mathrm{C} 11 \mathrm{~B}$ & $118.6(3)$ \\
\hline $\mathrm{C} 15 \mathrm{~A}-\mathrm{C} 10 \mathrm{~A}-\mathrm{C} 9 \mathrm{~A}$ & $122.1(3)$ & $\mathrm{C} 15 \mathrm{~B}-\mathrm{C} 10 \mathrm{~B}-\mathrm{C} 9 \mathrm{~B}$ & $118.7(2)$ \\
\hline $\mathrm{C} 11 \mathrm{~A}-\mathrm{C} 10 \mathrm{~A}-\mathrm{C} 9 \mathrm{~A}$ & $118.6(2)$ & $\mathrm{C} 11 \mathrm{~B}-\mathrm{C} 10 \mathrm{~B}-\mathrm{C} 9 \mathrm{~B}$ & $122.7(2)$ \\
\hline $\mathrm{C} 12 \mathrm{~A}-\mathrm{C} 11 \mathrm{~A}-\mathrm{C} 10 \mathrm{~A}$ & $118.4(3)$ & $\mathrm{C} 12 \mathrm{~B}-\mathrm{C} 11 \mathrm{~B}-\mathrm{C} 10 \mathrm{~B}$ & $118.6(3)$ \\
\hline $\mathrm{C} 12 \mathrm{~A}-\mathrm{C} 11 \mathrm{~A}-\mathrm{H} 11 \mathrm{~A}$ & 120.8 & $\mathrm{C} 12 \mathrm{~B}-\mathrm{C} 11 \mathrm{~B}-\mathrm{H} 11 \mathrm{~B}$ & 120.7 \\
\hline $\mathrm{C} 10 \mathrm{~A}-\mathrm{C} 11 \mathrm{~A}-\mathrm{H} 11 \mathrm{~A}$ & 120.8 & $\mathrm{C} 10 \mathrm{~B}-\mathrm{C} 11 \mathrm{~B}-\mathrm{H} 11 \mathrm{~B}$ & 120.7 \\
\hline $\mathrm{F} 1 \mathrm{~A}-\mathrm{C} 12 \mathrm{~A}-\mathrm{C} 11 \mathrm{~A}$ & $118.2(3)$ & $\mathrm{F} 1 \mathrm{~B}-\mathrm{C} 12 \mathrm{~B}-\mathrm{C} 11 \mathrm{~B}$ & $118.3(3)$ \\
\hline $\mathrm{F} 1 \mathrm{~A}-\mathrm{C} 12 \mathrm{~A}-\mathrm{C} 13 \mathrm{~A}$ & $118.3(3)$ & $\mathrm{F} 1 \mathrm{~B}-\mathrm{C} 12 \mathrm{~B}-\mathrm{C} 13 \mathrm{~B}$ & $117.7(3)$ \\
\hline $\mathrm{C} 11 \mathrm{~A}-\mathrm{C} 12 \mathrm{~A}-\mathrm{C} 13 \mathrm{~A}$ & $123.5(3)$ & $\mathrm{C} 11 \mathrm{~B}-\mathrm{C} 12 \mathrm{~B}-\mathrm{C} 13 \mathrm{~B}$ & $123.9(3)$ \\
\hline $\mathrm{C} 12 \mathrm{~A}-\mathrm{C} 13 \mathrm{~A}-\mathrm{C} 14 \mathrm{~A}$ & $117.6(3)$ & $\mathrm{C} 12 \mathrm{~B}-\mathrm{C} 13 \mathrm{~B}-\mathrm{C} 14 \mathrm{~B}$ & $117.3(3)$ \\
\hline $\mathrm{C} 12 \mathrm{~A}-\mathrm{C} 13 \mathrm{~A}-\mathrm{H} 13 \mathrm{~A}$ & 121.2 & $\mathrm{C} 12 \mathrm{~B}-\mathrm{C} 13 \mathrm{~B}-\mathrm{H} 13 \mathrm{~B}$ & 121.3 \\
\hline $\mathrm{C} 14 \mathrm{~A}-\mathrm{C} 13 \mathrm{~A}-\mathrm{H} 13 \mathrm{~A}$ & 121.2 & $\mathrm{C} 14 \mathrm{~B}-\mathrm{C} 13 \mathrm{~B}-\mathrm{H} 13 \mathrm{~B}$ & 121.3 \\
\hline $\mathrm{C} 13 \mathrm{~A}-\mathrm{C} 14 \mathrm{~A}-\mathrm{C} 15 \mathrm{~A}$ & $121.1(3)$ & $\mathrm{C} 13 \mathrm{~B}-\mathrm{C} 14 \mathrm{~B}-\mathrm{C} 15 \mathrm{~B}$ & $120.8(3)$ \\
\hline $\mathrm{C} 13 \mathrm{~A}-\mathrm{C} 14 \mathrm{~A}-\mathrm{H} 14 \mathrm{~A}$ & 119.5 & $\mathrm{C} 13 \mathrm{~B}-\mathrm{C} 14 \mathrm{~B}-\mathrm{H} 14 \mathrm{~B}$ & 119.6 \\
\hline $\mathrm{C} 15 \mathrm{~A}-\mathrm{C} 14 \mathrm{~A}-\mathrm{H} 14 \mathrm{~A}$ & 119.5 & $\mathrm{C} 15 \mathrm{~B}-\mathrm{C} 14 \mathrm{~B}-\mathrm{H} 14 \mathrm{~B}$ & 119.6 \\
\hline $\mathrm{C} 14 \mathrm{~A}-\mathrm{C} 15 \mathrm{~A}-\mathrm{C} 10 \mathrm{~A}$ & $120.1(3)$ & $\mathrm{C} 14 \mathrm{~B}-\mathrm{C} 15 \mathrm{~B}-\mathrm{C} 10 \mathrm{~B}$ & $120.8(3)$ \\
\hline $\mathrm{C} 14 \mathrm{~A}-\mathrm{C} 15 \mathrm{~A}-\mathrm{H} 15 \mathrm{~A}$ & 119.9 & $\mathrm{C} 14 \mathrm{~B}-\mathrm{C} 15 \mathrm{~B}-\mathrm{H} 15 \mathrm{~B}$ & 119.6 \\
\hline $\mathrm{C} 10 \mathrm{~A}-\mathrm{C} 15 \mathrm{~A}-\mathrm{H} 15 \mathrm{~A}$ & 119.9 & $\mathrm{C} 10 \mathrm{~B}-\mathrm{C} 15 \mathrm{~B}-\mathrm{H} 15 \mathrm{~B}$ & 119.6 \\
\hline
\end{tabular}




$\begin{array}{ll}\mathrm{C} 6 \mathrm{~A}-\mathrm{C} 1 \mathrm{~A}-\mathrm{C} 2 \mathrm{~A}-\mathrm{C} 3 \mathrm{~A} & -0.2(5) \\ \mathrm{C} 1 \mathrm{~A}-\mathrm{C} 2 \mathrm{~A}-\mathrm{C} 3 \mathrm{~A}-\mathrm{C} 4 \mathrm{~A} & 0.2(5) \\ \mathrm{C} 1 \mathrm{~A}-\mathrm{C} 2 \mathrm{~A}-\mathrm{C} 3 \mathrm{~A}-\mathrm{B} 1 \mathrm{~A} & -179.4(2) \\ \mathrm{C} 2 \mathrm{~A}-\mathrm{C} 3 \mathrm{~A}-\mathrm{C} 4 \mathrm{~A}-\mathrm{C} 5 \mathrm{~A} & 0.1(5) \\ \mathrm{B} 1 \mathrm{~A}-\mathrm{C} 3 \mathrm{~A}-\mathrm{C} 4 \mathrm{~A}-\mathrm{C} 5 \mathrm{~A} & 179.6(2) \\ \mathrm{C} 3 \mathrm{~A}-\mathrm{C} 4 \mathrm{~A}-\mathrm{C} 5 \mathrm{~A}-\mathrm{C} 6 \mathrm{~A} & -0.3(5) \\ \mathrm{C} 4 \mathrm{~A}-\mathrm{C} 5 \mathrm{~A}-\mathrm{C} 6 \mathrm{~A}-\mathrm{C} 1 \mathrm{~A} & 0.3(5) \\ \mathrm{C} 4 \mathrm{~A}-\mathrm{C} 5 \mathrm{~A}-\mathrm{C} 6 \mathrm{~A}-\mathrm{C} 7 \mathrm{~A} & 179.3(3) \\ \mathrm{C} 2 \mathrm{~A}-\mathrm{C} 1 \mathrm{~A}-\mathrm{C} 6 \mathrm{~A}-\mathrm{C} 5 \mathrm{~A} & 0.0(4) \\ \mathrm{C} 2 \mathrm{~A}-\mathrm{C} 1 \mathrm{~A}-\mathrm{C} 6 \mathrm{~A}-\mathrm{C} 7 \mathrm{~A} & -179.0(3) \\ \mathrm{C} 5 \mathrm{~A}-\mathrm{C} 6 \mathrm{~A}-\mathrm{C} 7 \mathrm{~A}-\mathrm{O} 1 \mathrm{~A} & -5.7(4) \\ \mathrm{C} 1 \mathrm{~A}-\mathrm{C} 6 \mathrm{~A}-\mathrm{C} 7 \mathrm{~A}-\mathrm{O} 1 \mathrm{~A} & 173.3(3) \\ \mathrm{C} 5 \mathrm{~A}-\mathrm{C} 6 \mathrm{~A}-\mathrm{C} 7 \mathrm{~A}-\mathrm{C} 8 \mathrm{~A} & 174.3(3) \\ \mathrm{C} 1 \mathrm{~A}-\mathrm{C} 6 \mathrm{~A}-\mathrm{C} 7 \mathrm{~A}-\mathrm{C} 8 \mathrm{~A} & -6.8(4) \\ \mathrm{O} 1 \mathrm{~A}-\mathrm{C} 7 \mathrm{~A}-\mathrm{C} 8 \mathrm{~A}-\mathrm{C} 9 \mathrm{~A} & 1.0(5) \\ \mathrm{C} 6 \mathrm{~A}-\mathrm{C} 7 \mathrm{~A}-\mathrm{C} 8 \mathrm{~A}-\mathrm{C} 9 \mathrm{~A} & -179.0(3) \\ \mathrm{C} 7 \mathrm{~A}-\mathrm{C} 8 \mathrm{~A}-\mathrm{C} 9 \mathrm{~A}-\mathrm{C} 10 \mathrm{~A} & -178.7(3) \\ \mathrm{C} 8 \mathrm{~A}-\mathrm{C} 9 \mathrm{~A}-\mathrm{C} 10 \mathrm{~A}-\mathrm{C} 15 \mathrm{~A} & 0.9(5) \\ \mathrm{C} 8 \mathrm{~A}-\mathrm{C} 9 \mathrm{~A}-\mathrm{C} 10 \mathrm{~A}-\mathrm{C} 11 \mathrm{~A} & -179.6(3) \\ \mathrm{C} 15 \mathrm{~A}-\mathrm{C} 10 \mathrm{~A}-\mathrm{C} 11 \mathrm{~A}-\mathrm{C} 12 \mathrm{~A} & 0.0(4) \\ \mathrm{C} 9 \mathrm{~A}-\mathrm{C} 10 \mathrm{~A}-\mathrm{C} 11 \mathrm{~A}-\mathrm{C} 12 \mathrm{~A} & -179.4(3) \\ \mathrm{C} 10 \mathrm{~A}-\mathrm{C} 11 \mathrm{~A}-\mathrm{C} 12 \mathrm{~A}-\mathrm{F} 1 \mathrm{~A} & 178.9(3) \\ \text { C10A-C11A-C12A-C13A } & -0.2(5) \\ \text { F1A-C12A-C13A-C14A } & -178.9(3) \\ \text { C11A-C12A-C13A-C14A } & 0.3(5) \\ \text { C12A-C13A-C14A-C15A } & -0.1(6) \\ \text { C13A-C14A-C15A-C10A } & -0.1(6) \\ \text { C11A-C10A-C15A-C14A } & 0.1(5) \\ \text { C9A-C10A-C15A-C14A } & 179.6(3)\end{array}$

$\begin{array}{ll}\mathrm{C} 6 \mathrm{~B}-\mathrm{C} 1 \mathrm{~B}-\mathrm{C} 2 \mathrm{~B}-\mathrm{C} 3 \mathrm{~B} & 0.3(5) \\ \mathrm{C} 1 \mathrm{~B}-\mathrm{C} 2 \mathrm{~B}-\mathrm{C} 3 \mathrm{~B}-\mathrm{C} 4 \mathrm{~B} & -0.9(5) \\ \mathrm{C} 1 \mathrm{~B}-\mathrm{C} 2 \mathrm{~B}-\mathrm{C} 3 \mathrm{~B}-\mathrm{B} 1 \mathrm{~B} & 177.4(2) \\ \mathrm{C} 2 \mathrm{~B}-\mathrm{C} 3 \mathrm{~B}-\mathrm{C} 4 \mathrm{~B}-\mathrm{C} 5 \mathrm{~B} & 0.8(6) \\ \mathrm{Br} 1 \mathrm{~B}-\mathrm{C} 3 \mathrm{~B}-\mathrm{C} 4 \mathrm{~B}-\mathrm{C} 5 \mathrm{~B} & -177.5(3) \\ \mathrm{C} 3 \mathrm{~B}-\mathrm{C} 4 \mathrm{~B}-\mathrm{C} 5 \mathrm{~B}-\mathrm{C} 6 \mathrm{~B} & -0.1(6) \\ \mathrm{C} 4 \mathrm{~B}-\mathrm{C} 5 \mathrm{~B}-\mathrm{C} 6 \mathrm{~B}-\mathrm{C} 1 \mathrm{~B} & -0.5(5) \\ \mathrm{C} 4 \mathrm{~B}-\mathrm{C} 5 \mathrm{~B}-\mathrm{C} 6 \mathrm{~B}-\mathrm{C} 7 \mathrm{~B} & -179.9(3) \\ \mathrm{C} 2 \mathrm{~B}-\mathrm{C} 1 \mathrm{~B}-\mathrm{C} 6 \mathrm{~B}-\mathrm{C} 5 \mathrm{~B} & 0.4(4) \\ \mathrm{C} 2 \mathrm{~B}-\mathrm{C} 1 \mathrm{~B}-\mathrm{C} 6 \mathrm{~B}-\mathrm{C} 7 \mathrm{~B} & 179.8(3) \\ \mathrm{C} 5 \mathrm{~B}-\mathrm{C} 6 \mathrm{~B}-\mathrm{C} 7 \mathrm{~B}-\mathrm{O} 1 \mathrm{~B} & 174.3(3) \\ \mathrm{C} 1 \mathrm{~B}-\mathrm{C} 6 \mathrm{~B}-\mathrm{C} 7 \mathrm{~B}-\mathrm{O} 1 \mathrm{~B} & -5.2(4) \\ \mathrm{C} 5 \mathrm{~B}-\mathrm{C} 6 \mathrm{~B}-\mathrm{C} 7 \mathrm{~B}-\mathrm{C} 8 \mathrm{~B} & -6.3(4) \\ \mathrm{C} 1 \mathrm{~B}-\mathrm{C} 6 \mathrm{~B}-\mathrm{C} 7 \mathrm{~B}-\mathrm{C} 8 \mathrm{~B} & 174.2(3) \\ \mathrm{O} 1 \mathrm{~B}-\mathrm{C} 7 \mathrm{~B}-\mathrm{C} 8 \mathrm{~B}-\mathrm{C} 9 \mathrm{~B} & 3.9(4) \\ \mathrm{C} 6 \mathrm{~B}-\mathrm{C} 7 \mathrm{~B}-\mathrm{C} 8 \mathrm{~B}-\mathrm{C} 9 \mathrm{~B} & -175.5(3) \\ \mathrm{C} 7 \mathrm{~B}-\mathrm{C} 8 \mathrm{~B}-\mathrm{C} 9 \mathrm{~B}-\mathrm{C} 10 \mathrm{~B} & -179.7(3) \\ \mathrm{C} 8 \mathrm{~B}-\mathrm{C} 9 \mathrm{~B}-\mathrm{C} 10 \mathrm{~B}-\mathrm{C} 15 \mathrm{~B} & 173.9(3) \\ \mathrm{C} 8 \mathrm{~B}-\mathrm{C} 9 \mathrm{~B}-\mathrm{C} 10 \mathrm{~B}-\mathrm{C} 11 \mathrm{~B} & -4.5(5) \\ \mathrm{C} 15 \mathrm{~B}-\mathrm{C} 10 \mathrm{~B}-\mathrm{C} 11 \mathrm{~B}-\mathrm{C} 12 \mathrm{~B} & -0.5(4) \\ \mathrm{C} 9 \mathrm{~B}-\mathrm{C} 10 \mathrm{~B}-\mathrm{C} 11 \mathrm{~B}-\mathrm{C} 12 \mathrm{~B} & 177.9(3) \\ \mathrm{C} 10 \mathrm{~B}-\mathrm{C} 11 \mathrm{~B}-\mathrm{C} 12 \mathrm{~B}-\mathrm{F} 1 \mathrm{~B} & -178.9(3) \\ \mathrm{C} 10 \mathrm{~B}-\mathrm{C} 11 \mathrm{~B}-\mathrm{C} 12 \mathrm{~B}-\mathrm{C} 13 \mathrm{~B} & 0.2(5) \\ \mathrm{F} 1 \mathrm{~B}-\mathrm{C} 12 \mathrm{~B}-\mathrm{C} 13 \mathrm{~B}-\mathrm{C} 14 \mathrm{~B} & 178.8(3) \\ \mathrm{C} 11 \mathrm{~B}-\mathrm{C} 12 \mathrm{~B}-\mathrm{C} 13 \mathrm{~B}-\mathrm{C} 14 \mathrm{~B} & -0.3(5) \\ \mathrm{C} 12 \mathrm{~B}-\mathrm{C} 13 \mathrm{~B}-\mathrm{C} 14 \mathrm{~B}-\mathrm{C} 15 \mathrm{~B} & 0.6(5) \\ \mathrm{C} 13 \mathrm{~B}-\mathrm{C} 14 \mathrm{~B}-\mathrm{C} 15 \mathrm{~B}-\mathrm{C} 10 \mathrm{~B} & -0.9(5) \\ \mathrm{C} 11 \mathrm{~B}-\mathrm{C} 10 \mathrm{~B}-\mathrm{C} 15 \mathrm{~B}-\mathrm{C} 14 \mathrm{~B} & 0.8(4) \\ \mathrm{C} 9 \mathrm{~B}-\mathrm{C} 10 \mathrm{~B}-\mathrm{C} 15 \mathrm{~B}-\mathrm{C} 14 \mathrm{~B} & -177.7(3) \\ & \end{array}$

Hydrogen-bond geometry $\left(A,{ }^{\circ}\right)$

\begin{tabular}{lllll}
\hline$D-\mathrm{H} \cdots A$ & $D-\mathrm{H}$ & $\mathrm{H} \cdots A$ & $D \cdots A$ & $D-\mathrm{H} \cdots A$ \\
\hline $\mathrm{C} 13 A-\mathrm{H} 13 A \cdots \mathrm{O} 1 B$ & 0.93 & 2.52 & $3.427(4)$ & 165 \\
$\mathrm{C} 9 A-\mathrm{H} 9 A A \cdots \mathrm{O} 1 A^{\mathrm{i}}$ & 0.93 & 2.52 & $3.362(4)$ & 151 \\
$\mathrm{C} 11 A-\mathrm{H} 11 A \cdots \mathrm{O} 1 A^{\mathrm{i}}$ & 0.93 & 2.45 & $3.294(4)$ & 151 \\
$\mathrm{C} 15 B-\mathrm{H} 15 B \cdots \mathrm{O} 1 B^{\mathrm{ii}}$ & 0.93 & 2.50 & $3.377(4)$ & 157 \\
\hline
\end{tabular}

Symmetry codes: (i) $-x+1,-y+1,-z+1$; (ii) $-x+1,-y+1,-z+2$. 